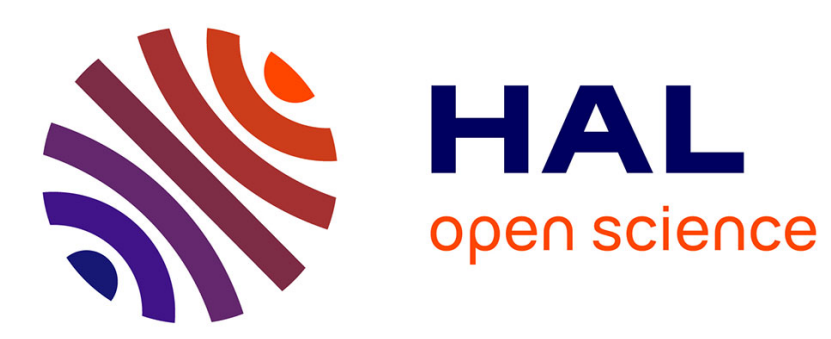

\title{
Extended Narrow Escape with Many Windows for Analyzing Viral Entry into the Cell Nucleus
}

\author{
Thibault Lagache, D. Holcman
}

\section{To cite this version:}

Thibault Lagache, D. Holcman. Extended Narrow Escape with Many Windows for Analyzing Viral Entry into the Cell Nucleus. Journal of Statistical Physics, 2017, 166 (2), pp.244-266. 10.1007/s10955016-1691-9 . hal-02911977

\section{HAL Id: hal-02911977 \\ https://hal.science/hal-02911977}

Submitted on 12 Aug 2020

HAL is a multi-disciplinary open access archive for the deposit and dissemination of scientific research documents, whether they are published or not. The documents may come from teaching and research institutions in France or abroad, or from public or private research centers.
L'archive ouverte pluridisciplinaire HAL, est destinée au dépôt et à la diffusion de documents scientifiques de niveau recherche, publiés ou non, émanant des établissements d'enseignement et de recherche français ou étrangers, des laboratoires publics ou privés. 


\title{
Extended Narrow Escape with ManyWindows for Analyzing Viral Entry into the Cell Nucleus
}

\author{
T. Lagache ${ }^{1,2}$ and D. Holcman ${ }^{1,3}$ *
}

August 12, 2020

\begin{abstract}
Many viruses must enter the cell nucleus through small nanopores in order to replicate. We model here viral motion as a stochastic process described by the survival Fokker-Planck equation. We estimate the probability and the conditional mean first passage time that a viral trajectory is absorbed at a small nuclear pore before being terminated. The method is based on the explicit Neuman-Green's function. The cell nucleus is model as a three dimensional ball, covered with thousands of small absorbing windows. The minimum distance between them defines the smallest spatial scale that is an unavoidable limit for efficient stochastic simulations. Derived asymptotic formula agree with stochastic simulations and reveal how small and large geometrical parameters define the cytoplasmic stage of viral infection.
\end{abstract}

\section{Introduction}

Understanding the biophysical properties of particles such as molecules, proteins, DNA, RNA, or viruses that are moving inside the crowded cellular environment [23] remains a challenge both experimentally and theoretically. For example, vesicles or RNA granules [10] have to reach small targets in order to deliver their payload or trigger protein synthesis. If small DNA or plasmid can pass the cytoplasmic crowded organization, the motion of larger ones is largerly impaired [9]. In some cases, large Brownian particles are transported intermittently along microtubules (MTs) inside the cytoplasm toward the nucleus. Many viruses containing DNA have the ability to hijack the cellular transport machinery to reach a nuclear pore and deliver their genetic material inside the nucleus [29, 11]. Viral

${ }^{* 1}$ Applied Mathematics and Computational Biology, Ecole Normale Supérieure, France. ${ }^{2}$ The NeuroTechnology Center at Columbia University Biological Sciences 901 NWC Building, 550 West 120th Street, New York, N.Y. 10027. tl2756@columbia.edu. ${ }^{3}$ Newton Institute, Churchill college and DAMTP Cambridge CB30DS, United Kingdom. 
trajectories can be monitored in vivo using live microscopy and for viruses such as Human Immunodeficiency Virus or the Adeno-Associated Virus [1, 27], these trajectories consist of alternating epochs of diffusion and directed motion. The precise driving force and physical nature of these trajectories remains unclear. In addition inside the cytoplasm, viruses can be trapped or degraded through several molecular pathways (including the ubiquitin-proteasome).

To quantify the success of the early steps of viral infection, we previously modeled single viral particle as individual stochastic processes $[12,17]$. Our goal was to estimate the success that a trajectory reaches a small window (nuclear pore) located on the nucleus surface (Fig. 1). However, due to small size of the nuclear pore, Brownian simulations are most of the time ineffective to estimate precisely the statistical moments associated with the arrival time distribution. Thus, to study the dependency with respect to geometrical and dynamical parameters, we analyzed the intermittent stochastic dynamics of viruses along MTs and derived asymptotic formula for the conditional mean first passage time (MFPT) $\langle\tau\rangle(n, \epsilon)$ and the probability $\langle P\rangle(n, \epsilon)$ that a single particle arrives to one over $n$ small targets with radius $\epsilon[12,19]$. However, these formula are valid when the number of absorbing holes (targets) is not too large and that holes are well separated. We shall extend here this previous analysis to the case of many small holes. This analysis relies on the explicit expansion of the Neumann-Green's function to order three [24], and is valid for many interacting small holes $[26,15,6]$.

In the limit of many absorbing holes distributed on a small spherical target inside a bounded domain (a biological round cell), we compute here a novel mean arrival time formula to an absorbing window, that falls into the Narrow escape time (NET) framework [16] . This computation clarifies the dependency of the NET with respect to the number of holes, their distribution, the radius of the spherical target and the percentage of the target surface covered by holes. This analysis extents the well-known homogenization formula obtained by Berg and Purcell for mixed Neumann-Dirichlet boundary conditions [3]. The paper is organized as follows. First, we recall our previous model of viral particles and the stochastic description of trajectories. Second, we extend the NET method to many absorbing holes and compute the mean arrival time formula using an interaction matrix between holes. Then, computing the Neumann's function around a small reflecting sphere, we derive asymptotic formula for the probability and the conditional mean time to reach one small window in the limit of a large number of absorbing windows . Finally, we confirm our analysis with Brownian simulations. The new formula improve our previous effort $[12,19]$ and can now be used to quantify more precisely the first steps of viral infection in cells. In completely independent line of thoughts, a similar NET formula was recently derived using matched asymptotics for Brownian motion [21]. 

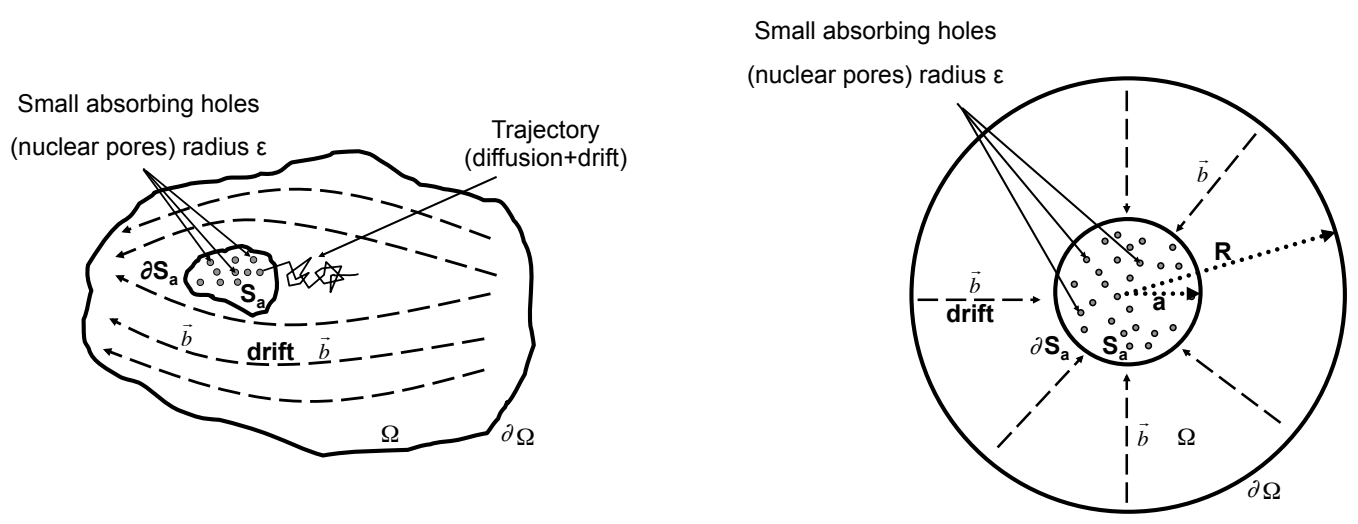

Figure 1: Schematic representation of the cell cytoplasm as a 3-dimensional domain $\Omega$. (Right): Stochastic trajectories, modeled by equation (2), with a diffusion and a drift terms. They can be absorbed at small windows with radius $\epsilon \ll|\Omega|^{1 / 3}$ located on the surface of the nucleus $\partial S_{a}$. (Left): simplified geometry: the cell geometry is a ball (radius $R$ ) containing a spherical nucleus surface, containing many small absorbing windows. The nucleus ball is of radius $a$ such that $\epsilon \ll a \ll|\Omega|^{1 / 3}$.

\section{Mean time to a small nuclear pore}

Intermittent trajectories of a viral particle $\mathbf{x}(t)$ are described as realization of the switching stochastic process [18]

$$
d \mathbf{x}=\left\{\begin{array}{lr}
\sqrt{2 D} d \mathbf{w} & \text { when } \quad \mathbf{x}(t) \quad \text { is free } \\
\mathbf{V} d t & \mathbf{x}(t) \quad \text { bound }
\end{array}\right.
$$

where $\mathbf{w}$ is a standard $3 \mathrm{~d}$-Brownian motion, $D$ the diffusion constant and $\mathbf{V}$ the velocity of the directed motion along MTs. The switching dynamics depends on the attachment and detachment rates [18]. In previous work [18, 19] we have coarse-grained this switching process into by a steady-state stochastic equation

$$
d \mathbf{x}=\mathbf{b}(\mathbf{x}) d t+\sqrt{2 D} d \mathbf{w}
$$

where the effective drift $\mathbf{b}(\mathbf{x})$ is calibrated using the following criteria: inside the cytoplasm $\Omega$, the mean first passage time of stochastic processes (1) and (2) is the same. The steadystate drift $\mathbf{b}(\mathbf{x})$ depends on the cell geometry, the number and distribution of MTs and the rates of binding and unbinding to MTs.

To replicate most viruses have to reach a small pore, modeled as an absorbing disk of radius $\epsilon \ll 1$ located on the boundary $\partial S_{a}$ of the nucleus. The external cell membrane defines the boundary $\partial \Omega$ for the stochastic process equation (2). The cell cytoplasm is 
represented as the three-dimensional bounded domain $\Omega$, whose boundary is $\partial \Omega \bigcup \partial S_{a}$. It consists of a reflecting part except on the boundary $\partial N_{a}$ which consists of $n$ small absorbing windows located on the nucleus (fig. 1-left).

We model the degradation activity in the cytoplasm by a steady-state killing rate $k(\mathbf{x})$ and a trajectory described by equation (2) can be terminated before reaching the absorbing boundary $\partial N_{a}$. The survival probability density function (SPDF) is the solution of the forward Fokker-Planck equation (FPE) [13]

$$
\begin{gathered}
\frac{\partial p(\mathbf{x}, t)}{\partial t}=\Delta p(\mathbf{x}, t)-\nabla \cdot \mathbf{b}(\mathbf{x}) p(\mathbf{x}, t)-k(\mathbf{x}) p(\mathbf{x}, t) \\
p(\mathbf{x}, 0)=p_{i}(\mathbf{x})
\end{gathered}
$$

with the boundary conditions:

$$
\begin{array}{r}
p(\mathbf{x}, t)=0 \text { on } \partial N_{a} \text { and } \\
\mathbf{J}(\mathbf{x}, t) \cdot \mathbf{n}_{\mathbf{x}}=0 \text { on } \partial \Omega \bigcup\left(\partial S_{a}-\partial N_{a}\right)
\end{array}
$$

where the flux density vector is

$$
\mathbf{J}(\mathbf{x}, t)=-D \nabla p(\mathbf{x}, t)+\mathbf{b}(\mathbf{x}) p(\mathbf{x}, t) .
$$

where $\mathbf{n}_{\mathbf{x}}$ is the normal vector at point $\mathbf{x}$.

We recall that the mean probability $\langle P\rangle$ and the conditional MFPT $\langle\tau\rangle$ (averaged over a uniform particle distribution) to reach the boundary $\partial N_{a}$ before termination is expressed using the total time spent at a point

$$
\tilde{p}(\mathbf{x})=\int_{0}^{\infty} p(\mathbf{x}, t) d t
$$

and

$$
q(\mathbf{x})=\int_{0}^{\infty} t p(\mathbf{x}, t) d t
$$

[12]. The expressions of the probability and arrival time with respect to the solution of the FPE are

$$
\langle P\rangle(n, \epsilon)=1-\int_{\Omega} k(\mathbf{x}) \tilde{p}(\mathbf{x}) d \mathbf{x}
$$

and

$$
\langle\tau\rangle(n, \epsilon)=\frac{\int_{\Omega} \tilde{p}(\mathbf{x}) d \mathbf{x}-\int_{\Omega} k(\mathbf{x}) q(\mathbf{x}) d \mathbf{x}}{1-\int_{\Omega} k(\mathbf{x}) \tilde{p}(\mathbf{x}) d \mathbf{x}} .
$$


For a drift $\mathbf{b}(\mathbf{x})=-\nabla \Phi(\mathbf{x})$, an asymptotic expansion with the small parameter $\epsilon$ reveals that $[12]$

$$
\left\{\begin{aligned}
\langle P\rangle(n, \epsilon)= & \frac{1}{\frac{1}{4 D n \epsilon} \int_{\Omega} e^{-\frac{\Phi(\mathbf{x})}{D}} k(\mathbf{x}) d \mathbf{x}+e^{-\frac{\Phi_{0}}{D}}} \\
\langle\tau\rangle(n, \epsilon)= & \frac{1}{\frac{1}{4 D n \epsilon} \int_{\Omega} e^{-\frac{\Phi(\mathbf{x})}{D}} d \mathbf{x}} \int_{\Omega}^{-\frac{\Phi(\mathbf{x})}{D}} k(\mathbf{x}) d \mathbf{x}+e^{-\frac{\Phi_{0}}{D}}
\end{aligned}\right.
$$

where $\Phi_{0}$ is the constant value of the radial potential $\Phi(\mathbf{x})$ on the centered nucleus where the nuclear pores are uniformly distributed. The range of validity of these asymptotic expressions has been explored with Brownian simulations for a single hole [17]. However, these formulas do not account for the possible interactions between the small absorbing pores, and for a large number of nuclear pores $n \gg 1$,

$$
\lim _{n \rightarrow \infty, n \epsilon^{2} \ll 1}\langle\tau\rangle(n, \epsilon)=0,
$$

which shows the limitation of the previous formula.

Early results demonstrated that holes' interactions can drastically affect the MFPT, especially when the distance between the holes tends to zero [26, 15]. Refined analysis using analytical expression of the Green's function in the unit disk, sphere or ball based on solving a linear system of equations for the fluxes at each window revealed how the MFPT depends on the position of the windows $[8,6,24,5]$. In particular, the optimal configuration of windows that minimizes the MFPT was related to the classical Fekete problem of minimizing the Coulomb energy on a ball $[8,6,24,7]$.

However, the linear system of equations for the flux at each window does not hold when windows are distributed on the surface of ball with a small radius (of the order of few window size). We shall derive here the correction term that accounts for the nuclear geometry. First, we derive the narrow escape time for a stochastic particle (with a drift) in the presence of a killing field $k(x)$ to reach an absorbing window. Second, by computing analytically the Neumann function for a reflecting ball, we derive a coupled system of equations for the fluxes at each interacting window covering the ball (spherical nucleus). Finally, for a large number of holes, we use a mean-field approximation and obtain formulas for the probability $\langle P\rangle$ and the mean time $\langle\tau\rangle$, valid for a large range of parameters $\epsilon$ (window size) and $n$, generalizing formula (11) and extending previous attempts to homogenize mixed Dirichlet-Neumann boundary conditions $[3,30,2,25]$. We summarize now the main asymptotic 
formula (86) and (87). We define the function

$$
F(n, a, \epsilon)=\frac{n a D \epsilon}{\left(\pi a+\epsilon \log \left(\frac{\epsilon}{a}\right)+n \epsilon\left(1-2 \alpha_{0}-\alpha_{0}^{2} \log \left(\alpha_{0}\right)\right)\right)}
$$

with

$$
\alpha_{0}=\left\{\begin{array}{lc}
\frac{\epsilon}{a} & \text { for uniformly randomly distributed windows, } \\
\frac{1}{\sqrt{n}} & \text { for regularly distributed windows. }
\end{array}\right.
$$

Using the same notations as in formula (11), when the drift is pointing towards the nucleus center and the potential $\Phi(\boldsymbol{x})=\Phi_{0}$ is constant at nuclear surface, the probability and MFPT formulas are

$$
\langle P\rangle=\frac{4 \pi F(n, a, \epsilon) e^{-\frac{\Phi_{0}}{D}}}{\int_{\Omega} k(\mathbf{x}) e^{-\frac{\phi(\mathbf{x})}{D}} d \mathbf{x}+4 \pi F(n, a, \epsilon) e^{-\frac{\Phi_{0}}{D}}},
$$

and

$$
\langle\tau\rangle=\frac{\int_{\Omega} e^{-\frac{\phi(\mathbf{x})}{D}} d \mathbf{x}}{\int_{\Omega} k(\mathbf{x}) e^{-\frac{\phi(\mathbf{x})}{D}} d \mathbf{x}+4 \pi F(n, a, \epsilon) e^{-\frac{\Phi_{0}}{D}} .}
$$

Finally, we test the robustness of our asymptotic formula against Brownian simulations and apply our formula to describe viral trafficking to a nuclear pore $(n \approx 2,000 \gg 1[\mathbf{2 2}])$ covering the nucleus.

\section{Asymptotic derivations of the mean time $\langle\tau\rangle$ and the probability $\langle P\rangle$}

The $n$-absorbing windows $\partial N_{a}=\bigcup_{i=1}^{n} \partial \Omega_{i}$ have the same radius $\epsilon$, centered at positions $\left(\mathbf{x}_{i}\right)_{i=1}^{n}$. The steady state SPDF $p$ is solution of equation (3) [13].

The associated Neumann-Green function $\mathcal{N}\left(\mathbf{x}, \mathbf{x}_{0}\right)$ is solution of the differential equation $[12]$

$$
\begin{aligned}
& \Delta \mathcal{N}\left(\mathbf{x}, \mathbf{x}_{0}\right)=-\delta_{\mathbf{x}_{0}}(\mathbf{x}), \mathbf{x} \in \Omega, \\
& \frac{\partial \mathcal{N}}{\partial n}\left(\mathbf{x}, \mathbf{x}_{0}\right)=-\frac{1}{|\partial \Omega|}, \text { for } \mathbf{x} \in \partial \Omega .
\end{aligned}
$$

We recall that $\tilde{p}(\mathbf{x})=\int_{0}^{\infty} p(\mathbf{x}, t) d t$ is solution of equation

$$
\Delta \tilde{p}(\mathbf{x})-\nabla \cdot(\mathbf{b}(\mathbf{x}) \tilde{p}(\mathbf{x}))-k(\mathbf{x}) \tilde{p}(\mathbf{x})=-p_{i}(\mathbf{x}),
$$


where $p_{i}(\mathbf{x})$ is the normalized probability density function for the initial point

$$
\int_{\Omega} p_{i}(\mathbf{x})=1
$$

The boundary conditions for equation (18) are given by

$$
\tilde{p}(\mathbf{x})=0 \text { on } \partial N_{a}=\bigcup_{i=1}^{n} \partial \Omega_{i}
$$

and

$$
\tilde{\mathbf{J}}(\mathbf{x}) \cdot \mathbf{n}_{\mathbf{x}}=0 \text { on } \partial \Omega \bigcup\left(\partial S_{a}-\partial N_{a}\right)
$$

where the steady-state probability flux is given by

$$
\tilde{\mathbf{J}}(\mathbf{x})=-D \nabla \tilde{p}(\mathbf{x})+\mathbf{b}(\mathbf{x}) \tilde{p}(\mathbf{x}) .
$$

We recall that the average arrival probability [12] is given by

$$
\langle P\rangle=\int_{\partial N_{a}} \tilde{\mathbf{J}}(\mathbf{x}) \cdot \mathbf{n}_{\mathbf{x}} d \boldsymbol{x}=1-\int_{\Omega} k(\mathbf{x}) \tilde{p}(\mathbf{x}) d \mathbf{x}
$$

Thus we shall compute here $\tilde{p}$. For this, we compute the following integral

$$
\begin{aligned}
& I=\int_{\Omega}(\Delta \tilde{p}(\mathbf{x})-\nabla \cdot(\mathbf{b}(\mathbf{x}) \tilde{p}(\mathbf{x}))-k(\mathbf{x}) \tilde{p}(\mathbf{x})) \mathcal{N}\left(\mathbf{x}, \mathbf{x}_{0}\right) d S_{\mathbf{x}} \\
& -\int_{\Omega} \Delta \mathcal{N}\left(\mathbf{x}, \mathbf{x}_{0}\right) \tilde{p}(\mathbf{x}) d \mathbf{x} .
\end{aligned}
$$

Using the definition of the Neumann-Green function (17) and that $\tilde{p}$ is solution of equation (18), we obtain

$$
I=-\int_{\Omega} p_{i}(\mathbf{x}) \mathcal{N}\left(\mathbf{x}, \mathbf{x}_{0}\right)+\tilde{p}\left(\mathbf{x}_{0}\right)
$$

Moreover, using the Green's identity:

$$
\begin{array}{r}
\int_{\Omega}\left(\mathcal{N}\left(\mathbf{x}, \mathbf{x}_{0}\right) \Delta \tilde{p}(\mathbf{x})-\tilde{p}(\mathbf{x}) \Delta \mathcal{N}\left(\mathbf{x}, \mathbf{x}_{0}\right)\right) d \mathbf{x}= \\
\int_{\partial \Omega}\left(\mathcal{N}\left(\mathbf{x}, \mathbf{x}_{0}\right) \frac{\partial \tilde{p}(\mathbf{x})}{\partial n}-\tilde{p}(\mathbf{x}) \frac{\partial \mathcal{N}\left(\mathbf{x}, \mathbf{x}_{0}\right)}{\partial n}\right) .
\end{array}
$$

we get another expression for I

$$
\begin{aligned}
I & =\int_{\partial \Omega}\left(\mathcal{N}\left(\mathbf{x}, \mathbf{x}_{0}\right) \frac{\partial \tilde{p}(\mathbf{x})}{\partial n}-\tilde{p}(\mathbf{x}) \frac{\partial \mathcal{N}\left(\mathbf{x}, \mathbf{x}_{0}\right)}{\partial n}\right) \\
& +\int_{\Omega}(-\nabla \cdot(\mathbf{b}(\mathbf{x}) \tilde{p}(\mathbf{x}))-k(\mathbf{x}) \tilde{p}(\mathbf{x})) \mathcal{N}\left(\mathbf{x}, \mathbf{x}_{0}\right) d \mathbf{x} .
\end{aligned}
$$


Using an integration by part, we have

$$
\begin{array}{r}
\int_{\Omega}(-\nabla \cdot(\mathbf{b}(\mathbf{x}) \tilde{p}(\mathbf{x}))) \mathcal{N}\left(\mathbf{x}, \mathbf{x}_{0}\right) d \mathbf{x}=\int_{\Omega}(\mathbf{b}(\mathbf{x}) \tilde{p}(\mathbf{x})) \nabla \mathcal{N}\left(\mathbf{x}, \mathbf{x}_{0}\right) d \mathbf{x} \\
-\int_{\partial \Omega}\left(\mathbf{b}(\mathbf{x}) \cdot n_{\mathbf{x}}\right) \tilde{p}(\mathbf{x}) \mathcal{N}\left(\mathbf{x}, \mathbf{x}_{0}\right) d S_{\mathbf{x}} .
\end{array}
$$

By definition of the boundary condition of Neumann-Green function $\mathcal{N}$,

$$
\int_{\partial \Omega} \tilde{p}(\mathbf{x}) \frac{\partial \mathcal{N}\left(\mathbf{x}, \mathbf{x}_{0}\right)}{\partial n} d S_{\mathbf{x}}=-\frac{1}{|\partial \Omega|} \int_{\partial \Omega} \tilde{p}(\mathbf{x}) d S_{\mathbf{x}}
$$

We conclude that

$$
\begin{aligned}
I & =-\int_{\partial N_{a}} \tilde{\mathbf{J}}(\mathbf{x}) \cdot \mathbf{n}_{\mathbf{x}} \mathcal{N}\left(\mathbf{x}, \mathbf{x}_{0}\right) d \mathbf{x}+\int_{\Omega} \mathbf{b}(\mathbf{x}) \cdot \nabla \mathcal{N}\left(\mathbf{x}, \mathbf{x}_{0}\right) \tilde{p}(\mathbf{x}) d \mathbf{x} \\
& -\int_{\Omega} k(\mathbf{x}) \tilde{p}(\mathbf{x}) \mathcal{N}\left(\mathbf{x}, \mathbf{x}_{0}\right) d \mathbf{x}+\frac{1}{|\partial \Omega|} \int_{\partial \Omega} \tilde{p}(\mathbf{x}) d S_{\mathbf{x}} .
\end{aligned}
$$

Finally, merging the two relations (32) and (25) for the integral $I$, we get the identity:

$$
\begin{aligned}
\int_{\Omega}\left(k(\mathbf{x}) \tilde{p}(\mathbf{x})-p_{i}(\mathbf{x})\right) \mathcal{N}\left(\mathbf{x}, \mathbf{x}_{0}\right) d \mathbf{x} & =-\int_{\partial N_{a}} \tilde{\mathbf{J}}(\mathbf{x}) \cdot \mathbf{n}_{\mathbf{x}} \mathcal{N}\left(\mathbf{x}, \mathbf{x}_{0}\right) d \mathbf{x} \\
& +\int_{\Omega} \mathbf{b}(\mathbf{x}) \cdot \nabla \mathcal{N}\left(\mathbf{x}, \mathbf{x}_{0}\right) \tilde{p}(\mathbf{x}) d \mathbf{x} \\
& +\frac{1}{|\partial \Omega|} \int_{\partial \Omega} \tilde{p}(\mathbf{x}) d \mathbf{x}-\tilde{p}\left(\mathbf{x}_{0}\right) .
\end{aligned}
$$

When the vector field is the gradient of a potential $\mathbf{b}=-\nabla \Phi$, we shall use the following approximation [12] for the solution

$$
\tilde{p}(\mathbf{x})=C_{\epsilon} e^{-\frac{\Phi(\mathbf{x})}{D}},
$$

where $C_{\epsilon}$ is a constant that depend on $\varepsilon$, which tends to $\infty$ when $\epsilon$ tends to 0 . We shall now only retain in relation (33) leading order terms in $\epsilon$. For a smooth initial distributions $p_{i}$, the integral

$$
\int_{\Omega} p_{i}(\mathbf{x}) \mathcal{N}\left(\mathbf{x}, \mathbf{x}_{0}\right) d \mathbf{x}=O(1)
$$

is uniformly bounded when $\epsilon \rightarrow 0$ (the proof was given in [14] by deriving the regular Laplace equation satisfied by the function $\left.\mathbf{x}_{0} \rightarrow \int_{\Omega} p_{i}(\mathbf{x}) \mathcal{N}\left(\mathbf{x}, \mathbf{x}_{0}\right) d \mathbf{x}\right)$. All other terms diverge. We shall also consider here the case of a small degradation rate $k(\mathbf{x}) \ll 1$. In 
that case, we will neglect the term $\int_{\Omega} k(\mathbf{x}) \tilde{p}(\mathbf{x}) \mathcal{N}\left(\mathbf{x}, \mathbf{x}_{0}\right) d \mathbf{x}$ compared to all other terms in (33). With these approximations, relation (33) can be written at leading order as

$$
\begin{aligned}
\tilde{p}\left(\mathbf{x}_{0}\right)+O(1) & =-\int_{\partial N_{a}} \tilde{\mathbf{J}}(\mathbf{x}) \cdot \mathbf{n}_{\mathbf{x}} \mathcal{N}\left(\mathbf{x}, \mathbf{x}_{0}\right) d S_{\mathbf{x}}+\int_{\Omega} \mathbf{b}(\mathbf{x}) \cdot \nabla \mathcal{N}\left(\mathbf{x}, \mathbf{x}_{0}\right) \tilde{p}(\mathbf{x}) d \mathbf{x} \\
& +\frac{1}{|\partial \Omega|} \int_{\partial \Omega} \tilde{p}(\mathbf{x}) d \mathbf{x} .
\end{aligned}
$$

For an initial point $\boldsymbol{x}_{0}$ at a distance $O(1)$ away from any absorbing window, the Neumann function $\mathcal{N}\left(\mathbf{x}, \mathbf{x}_{0}\right)$ is uniformly bounded for $\boldsymbol{x} \in \partial \Omega_{a}$. In addition, integrating relation (18) over $\Omega$, we obtain

$$
\int_{\partial N_{a}} \tilde{\mathbf{J}}(\mathbf{x}) \cdot \mathbf{n}_{\mathbf{x}} d S \boldsymbol{x}=1-\int_{\Omega} k(\mathbf{x}) \tilde{p}(\mathbf{x}) d \mathbf{x}=\langle P\rangle \in[0,1] .
$$

Thus for $\mathbf{x} \in \partial N_{a}$ the absorbing boundary, the flux $\tilde{\mathbf{J}}(\mathbf{x}) \cdot \mathbf{n}_{\mathbf{x}}=-D \frac{\partial \tilde{p}(\mathbf{x})}{\partial n_{\mathbf{x}}}>0$ and thus the integral $\int_{\partial N_{a}} \tilde{\mathbf{J}}(\mathbf{x}) \cdot \mathbf{n}_{\mathbf{x}} \mathcal{N}\left(\mathbf{x}, \mathbf{x}_{0}\right) d S_{\mathbf{x}}$ is uniformly bounded in $\mathbf{x}_{0} \in \Omega$. Thus for $\boldsymbol{x}_{0}$ at a distance $O(1)$ away from the absorbing windows, relation (36) can be written

$$
\frac{1}{|\partial \Omega|} \int_{\partial \Omega} \tilde{p}(\mathbf{x}) d \mathbf{x}+\int_{\Omega} \mathbf{b}(\mathbf{x}) \cdot \nabla \mathcal{N}\left(\mathbf{x}, \mathbf{x}_{0}\right) \tilde{p}(\mathbf{x}) d \mathbf{x}=\tilde{p}\left(\mathbf{x}_{0}\right)
$$

which is the integral solution $\tilde{p}\left(\mathbf{x}_{0}\right)=C_{\epsilon} e^{-\frac{\Phi\left(\mathbf{x}_{0}\right)}{D}}$ for the Fokker-Planck equation with no absorbing windows.

$$
\frac{1}{|\partial \Omega|} \int_{\partial \Omega} \tilde{p}(\mathbf{x}) d \mathbf{x}+\int_{\Omega} \mathbf{b}(\mathbf{x}) \cdot \nabla \mathcal{N}\left(\mathbf{x}, \mathbf{x}_{0}\right) \tilde{p}(\mathbf{x}) d \mathbf{x}=C_{\epsilon} e^{-\frac{\Phi\left(\mathbf{x}_{0}\right)}{D}}+O(1) .
$$

We shall now take $\boldsymbol{y} \in \partial N_{a}$ in relation (36)

$$
\begin{aligned}
0 & =-\int_{\partial N_{a}} \tilde{\mathbf{J}}(\mathbf{x}) \cdot \mathbf{n}_{\mathbf{x}} \mathcal{N}(\mathbf{x}, \mathbf{y}) d S_{\mathbf{x}}+\int_{\Omega} \mathbf{b}(\mathbf{x}) \cdot \nabla \mathcal{N}(\mathbf{x}, \mathbf{y}) \tilde{p}(\mathbf{x}) d \mathbf{x} \\
& +\frac{1}{|\partial \Omega|} \int_{\partial \Omega} \tilde{p}(\mathbf{x}) d \mathbf{x} .
\end{aligned}
$$

Using relation (39), we finally obtain

$$
0=-\int_{\partial N_{a}} \tilde{\mathbf{J}}(\mathbf{x}) \cdot \mathbf{n}_{\mathbf{x}} \mathcal{N}\left(\mathbf{x}, \mathbf{x}_{0}\right) d \mathbf{x}+C_{\epsilon} e^{-\frac{\Phi\left(\mathbf{x}_{0}\right)}{D}}(1+o(1)),
$$

(because we removed in the two integrals a boundary layer near the absorbing windows). We shall now compute $\int_{\partial N_{a}} \tilde{\mathbf{J}}(\mathbf{x}) \cdot \mathbf{n}_{\mathbf{x}} \mathcal{N}\left(\mathbf{x}, \mathbf{x}_{0}\right) d \mathbf{x}=\sum_{i=1}^{n} \int_{\partial \Omega_{i}} \tilde{\mathbf{J}}(\mathbf{x}) \cdot \mathbf{n}_{\mathbf{x}} \mathcal{N}\left(\mathbf{x}, \mathbf{x}_{0}\right) d \mathbf{x}$, by decomposing the flux

$$
\left(\tilde{\mathbf{J}}(\mathbf{x}) \cdot \mathbf{n}_{\mathbf{x}}\right)_{\boldsymbol{x} \in \partial \Omega_{i}}=g_{i}(\boldsymbol{x})+f_{i}(\boldsymbol{x})
$$


where the leading order $g_{i}(s)$ with

$$
s=\left|\mathbf{x}-\mathbf{x}_{i}\right|
$$

into

$$
g_{i}(s)=\frac{g_{0}^{i}}{\sqrt{\epsilon^{2}-s^{2}}}
$$

and $g_{0}^{i}$ a constant and $f_{i}$ is a regular function such that

$$
\int_{0}^{\epsilon} f_{i}(s) d s=O\left(\epsilon g_{0}^{i}\right)
$$

Choosing $\boldsymbol{y}=\boldsymbol{x}_{i}$ at the center of each absorbing windows, we get that $\tilde{p}\left(\mathbf{x}_{i}\right)=0$. For $i \neq j$, and $\left|\mathbf{x}_{i}-\mathbf{x}_{j}\right| \gg \epsilon$ and for $\mathbf{x} \in \partial \Omega_{j}$,

$$
\mathcal{N}\left(\mathbf{x}, \mathbf{x}_{i}\right)=\mathcal{N}\left(\mathbf{x}_{j}, \mathbf{x}_{i}\right)+O(\epsilon) .
$$

Consequently, using the flux expansion (42), we get

$$
\begin{aligned}
\int_{\partial N_{a}} \tilde{\mathbf{J}}(\mathbf{x}) \cdot \mathbf{n}_{\mathbf{x}} \mathcal{N}\left(\mathbf{x}, \mathbf{x}_{i}\right) d \mathbf{x} & =\int_{\partial \Omega_{i}}\left(g_{i}(\boldsymbol{x})+f_{i}(\boldsymbol{x})\right) \mathcal{N}\left(\mathbf{x}, \mathbf{x}_{i}\right) d \mathbf{x} \\
& +\sum_{j=1, j \neq i}^{n}\left(\mathcal{N}\left(\mathbf{x}_{\mathbf{j}}, \mathbf{x}_{i}\right)+0(\epsilon)\right) \int_{\partial \Omega_{j}}\left(g_{j}(\boldsymbol{x})+f_{j}(\boldsymbol{x})\right) d \mathbf{x} .
\end{aligned}
$$

For $\mathbf{x}_{i} \in \partial S_{a}$, the Neumann-Green's function $\mathcal{N}\left(\mathbf{x}, \mathbf{x}_{i}\right)$ can be written as [28]:

$$
\mathcal{N}\left(\mathbf{x}, \mathbf{x}_{i}\right)=\frac{1}{2 \pi D\left|\mathbf{x}-\mathbf{x}_{i}\right|}+\frac{L\left(\boldsymbol{x}_{i}\right)+N\left(\boldsymbol{x}_{i}\right)}{8 \pi D} \log \left(\frac{1}{\left|\boldsymbol{x}-\boldsymbol{x}_{i}\right|}\right)+\omega_{\mathbf{x}_{i}}(\mathbf{x}),
$$

where $L\left(\boldsymbol{x}_{i}\right)$ and $N\left(\boldsymbol{x}_{i}\right)$ are the principal curvatures of $\partial S_{a}$ at $\boldsymbol{x}_{i}$ and $\omega_{\mathbf{x}_{i}}(\boldsymbol{x})$ is the regular part of the Green function, which is bounded for $\boldsymbol{x}$ in $\Omega$. However, when the absorbing small patches are located on the boundary of a small ball of radius $a$, expansion (48) is

not sufficient because the second term $\frac{-1}{4 \pi a D} \log \left(\frac{1}{\left|\boldsymbol{x}_{i}-\boldsymbol{x}_{j}\right|}\right)$ become much larger than the first term $\frac{1}{2 \pi D\left|\boldsymbol{x}_{i}-\boldsymbol{x}_{j}\right|}$ when $\left|\boldsymbol{x}_{i}-\boldsymbol{x}_{j}\right| \approx a$, and $a \ll|\Omega|^{\frac{1}{3}}$. A different expansion is needed that we shall now discuss.

\subsection{Computing the flux at small absorbing windows located on a small internal ball}

The solution of the Neumann's equation

$$
\begin{aligned}
& D \Delta \tilde{\mathcal{N}}\left(\boldsymbol{x}, \boldsymbol{x}_{0}\right)=-\delta\left(\boldsymbol{x}-\boldsymbol{x}_{0}\right), \text { for } \boldsymbol{x} \in \mathbb{R}^{3} \\
& D \frac{\partial \tilde{\mathcal{N}}}{\partial n}\left(\boldsymbol{x}, \boldsymbol{x}_{0}\right)=0, \text { for } \boldsymbol{x} \in S_{a} .
\end{aligned}
$$


is equal for $\left|\boldsymbol{x}_{0}\right|=|\boldsymbol{x}|=a$ to (see appendix)

$$
\tilde{\mathcal{N}}\left(\boldsymbol{x}, \boldsymbol{x}_{0}\right)=\frac{1}{2 \pi D\left|\boldsymbol{x}-\boldsymbol{x}_{0}\right|}+\frac{1}{4 \pi a D} \log \left(\frac{\left|\boldsymbol{x}-\boldsymbol{x}_{0}\right|}{2 a+\left|\boldsymbol{x}-\boldsymbol{x}_{0}\right|}\right) .
$$

Thus for $\boldsymbol{x}$ and $\boldsymbol{x}_{0}$ in the neighborhood of the sphere $S_{a}$, we have

$$
\mathcal{N}\left(\boldsymbol{x}, \boldsymbol{x}_{0}\right)=\tilde{\mathcal{N}}\left(\boldsymbol{x}, \boldsymbol{x}_{0}\right)+O(1) .
$$

Consequently, using the geodesic distance $s=d\left(P, \boldsymbol{x}_{i}\right)$, the flux term in relation (47) can now be computed

$$
\begin{aligned}
\int_{\partial N_{a}} \tilde{\mathbf{J}}(\mathbf{x}) \cdot \mathbf{n}_{\mathbf{x}} \mathcal{N}\left(\mathbf{x}, \mathbf{x}_{i}\right) d \mathbf{x}= & \int_{0}^{\epsilon}\left(\frac{g_{0}^{i}}{\sqrt{\epsilon^{2}-s^{2}}}+f_{i}(s)\right) \\
& \left(\frac{1}{2 \pi D s}+\frac{1}{4 \pi a D} \log \left(\frac{s}{2 a+s}\right)+O(1)\right) 2 \pi s d s \\
+ & \sum_{j=1, j \neq i}^{n}\left(\mathcal{N}\left(\mathbf{x}_{j}, \mathbf{x}_{i}\right)+0(\epsilon)\right) \int_{0}^{\epsilon}\left(\frac{g_{0}^{j}}{\sqrt{\epsilon^{2}-s^{2}}}+f_{j}(s)\right) 2 \pi s d s .
\end{aligned}
$$

Using condition (45), we obtain:

$$
\begin{aligned}
\int_{\partial N_{a}} \tilde{\mathbf{J}}(\mathbf{x}) \cdot \mathbf{n}_{\mathbf{x}} \mathcal{N}\left(\mathbf{x}, \mathbf{x}_{0}\right) d \mathbf{x} & =\frac{g_{0}^{i}}{D}\left(\frac{\pi}{2}+\frac{\epsilon}{2 a} \log \left(\frac{\epsilon}{a}\right)+O(\epsilon)\right) \\
& \left.+2 \pi \epsilon \sum_{j=1, j \neq i}^{n} \mathcal{N}\left(\mathbf{x}_{j}, \mathbf{x}_{i}\right) g_{0}^{j}(1+O(\epsilon))\right) .
\end{aligned}
$$

We recall that the constant $g_{0}^{i}$ is of order $g_{0}^{i}=O\left(\frac{1}{n \epsilon}\right)$, and that

$$
\mathcal{N}\left(\mathbf{x}_{j}, \mathbf{x}_{i}\right)=O\left(\frac{1}{\left|\boldsymbol{x}_{i}-\boldsymbol{x}_{j}\right|}\right)=O\left(\frac{1}{a}\right),
$$

thus we rewrite the flux condition as

$$
\begin{aligned}
\int_{\partial N_{a}} \tilde{\mathbf{J}}(\mathbf{x}) \cdot \mathbf{n}_{\mathbf{x}} \mathcal{N}\left(\mathbf{x}, \mathbf{x}_{0}\right) d \mathbf{x} & =\frac{g_{0}^{i}}{D}\left(\frac{\pi}{2}+\frac{\epsilon}{2 a} \log \left(\frac{\epsilon}{a}\right)\right) \\
& +2 \pi \epsilon \sum_{j=1, j \neq i}^{n} \mathcal{N}\left(\mathbf{x}_{j}, \mathbf{x}_{i}\right) g_{0}^{j}+O\left(\frac{\epsilon}{a}\right)+O\left(\frac{1}{n}\right)
\end{aligned}
$$

Injecting (56) in (41), for $\boldsymbol{y}=\boldsymbol{x}_{i}$, we obtain the system of $\mathrm{n}$ equations for the $\mathrm{n}+1$ variables $\left(g_{0}^{1}, . . g_{0}^{n}, C_{\epsilon}\right)$ :

$$
\left(\frac{\pi}{2 D}+\frac{\epsilon}{2 a D} \log \left(\frac{\epsilon}{a}\right)\right) g_{0}^{i}+2 \pi \epsilon \sum_{j=1, j \neq i}^{n} \mathcal{N}\left(\mathbf{x}_{j}, \mathbf{x}_{i}\right) g_{0}^{j}=C_{\epsilon} e^{-\frac{\Phi\left(\mathbf{x}_{i}\right)}{D}}+O(1) .
$$


To close the system of equation, we use the compatibility condition (37) with expression (42) and approximation (34) for the function $\tilde{p}$ :

$$
2 \pi \epsilon \sum_{i=1}^{n} g_{0}^{i}=1-C_{\epsilon} \int_{\Omega} k(\mathbf{x}) e^{-\frac{\Phi(\mathbf{x})}{D}} d \mathbf{x}+O(1)
$$

Finally, we obtain a linear system of $n+1$ equations (56-57) for the flux constant $g_{0}^{i}$, $i \leq i \leq n$ and for the constant $C_{\epsilon}$ summarized here:

$$
\left\{\begin{array}{l}
\frac{\pi}{2 D}+\frac{\epsilon}{2 a D} \log \left(\frac{\epsilon}{a}\right) g_{0}^{i}+2 \pi \epsilon \sum_{j=1, j \neq i}^{n} \mathcal{N}\left(\mathbf{x}_{j}, \mathbf{x}_{i}\right) g_{0}^{j}=C_{\epsilon} e^{-\frac{\Phi\left(\mathbf{x}_{i}\right)}{D}}+O(1), \text { for } 1 \leq i \leq n \\
2 \pi \epsilon \sum_{i=1}^{n} g_{0}^{i}=1-C_{\epsilon} \int_{\Omega} k(\mathbf{x}) e^{-\frac{\Phi(\mathbf{x})}{D}} d \mathbf{x}+O(1)
\end{array}\right.
$$

We will now obtain asymptotic estimates for $C_{\epsilon},\langle P\rangle$ and $\langle\tau\rangle$, by solving the linear system of equations (58) in the small $\epsilon$ limit. We recall the expressions for probability (34) and the arrival time:

$$
\langle P\rangle=1-C_{\epsilon} \int_{\Omega} k(\mathbf{x}) e^{-\frac{\Phi(\mathbf{x})}{D}} d \mathbf{x} \text { and }\langle\tau\rangle=C_{\epsilon} \int_{\Omega} e^{-\frac{\Phi(\mathbf{x})}{D}} d \boldsymbol{x}
$$

where this last identity is derived in the small killing rate limit $|k(\boldsymbol{x})| \ll 1[12]$. In the next section, we derive the asymptotic expressions for quantities in (59).

\section{Mean field approximation and asymptotics formula for $\langle\tau\rangle$ and $\langle P\rangle$ for $n \gg \frac{1}{\epsilon}$}

We derive now expressions for $\langle P\rangle$ and $\langle\tau\rangle$ in the limit $n \gg 1$ and absorbing windows are distributed with respect to a density $\rho(\boldsymbol{x})$ over the spherical nucleus $S_{a}$. By summing equation (56) for $1 \leq i \leq n$, we obtain that

$$
\left(\frac{\pi}{2 D}+\frac{\epsilon}{2 a D} \log \left(\frac{\epsilon}{a}\right)\right) \sum_{i=1}^{n} g_{0}^{i}+2 \pi \epsilon \sum_{i=1}^{n} g_{0}^{i} \sum_{j=1, j \neq i}^{n} \mathcal{N}\left(\mathbf{x}_{j}, \mathbf{x}_{i}\right)=C_{\epsilon} \sum_{i=1}^{n} e^{-\frac{\Phi\left(\mathbf{x}_{i}\right)}{D}}+O(n) .
$$

When $\boldsymbol{x}_{i}$ is located at the north pole, the distance $\left|\boldsymbol{x}_{i}-\boldsymbol{x}_{j}\right|$ with $j^{\text {th }}$ located at position $\boldsymbol{x}_{j}(\theta, \phi)$ is given by $\left|\boldsymbol{x}_{i}-\boldsymbol{x}_{j}\right|=2 a \sin \left(\frac{\phi}{2}\right)$ and the Neumann function is

$$
\tilde{\mathcal{N}}\left(\boldsymbol{x}_{j}(\theta, \phi), \boldsymbol{x}_{i}\right)=\frac{1}{4 \pi a D}\left(\frac{1}{\sin \left(\frac{\phi}{2}\right)}+\log \left(\frac{\sin \left(\frac{\phi}{2}\right)}{1+\sin \left(\frac{\phi}{2}\right)}\right)\right) \text {. }
$$


We use now that the probability density function $\rho_{i}(\phi)$ of the $j \neq i$ windows (north pole $i)$ is normalized

$$
\int_{0}^{\pi} 2 \pi a^{2} \rho_{i}(\phi) \sin (\phi) d \phi=1
$$

thus

$$
\begin{aligned}
\lim _{n \rightarrow \infty} \frac{1}{n} \sum_{j=1, j \neq i}^{n} \mathcal{N}\left(\mathbf{x}_{j}, \mathbf{x}_{i}\right) & =\int_{0}^{\pi} \frac{1}{4 \pi a D}\left(\frac{1}{\sin \left(\frac{\phi}{2}\right)}+\log \left(\frac{\sin \left(\frac{\phi}{2}\right)}{1+\sin \left(\frac{\phi}{2}\right)}\right)\right) \rho_{i}(\phi) 2 \pi a^{2} \sin (\phi) d \phi \\
& =\frac{a}{2 D} \int_{0}^{\pi}\left(\frac{1}{\sin \left(\frac{\phi}{2}\right)}+\log \left(\frac{\sin \left(\frac{\phi}{2}\right)}{1+\sin \left(\frac{\phi}{2}\right)}\right)\right) \rho_{i}(\phi) \sin (\phi) d \phi . \quad(63)
\end{aligned}
$$

In addition, we have the limit of the partial sum

$$
\lim _{n \rightarrow \infty} \frac{1}{n} \sum_{i=1}^{n} e^{-\frac{\Phi\left(\mathbf{x}_{i}\right)}{D}}=a^{2} \int_{0}^{2 \pi} \int_{0}^{\pi} e^{-\frac{\Phi(\phi, \theta)}{D}} \rho(\phi) d \phi d \theta,
$$

We now re-write relation (60) using the two integrals

$$
\begin{gathered}
I_{1}^{i}=\int_{0}^{\pi}\left(\frac{1}{\sin \left(\frac{\phi}{2}\right)}+\log \left(\frac{\sin \left(\frac{\phi}{2}\right)}{1+\sin \left(\frac{\phi}{2}\right)}\right)\right) \rho_{i}(\phi) \sin (\phi) d \phi \\
I_{2}=\int_{0}^{2 \pi} \int_{0}^{\pi} e^{-\frac{\Phi(\phi, \theta)}{D}} \rho(\phi, \theta) \sin (\phi) d \phi d \theta
\end{gathered}
$$

so that

$$
\left(\frac{\pi}{2 D}+\frac{\epsilon}{2 a D} \log \left(\frac{\epsilon}{a}\right)\right) \sum_{i=1}^{n} g_{0}^{i}+\frac{n a \pi \epsilon}{D} \sum_{i=1}^{n} g_{0}^{i} I_{1}^{i}=C_{\epsilon} n a^{2} I_{2}+O(n) .
$$

For identically distributed windows $1 \leq i \leq n, I_{1}^{i}=I_{1}$. Using the compatibility condition (57) in equation (67), we obtain

$$
\left(\frac{1}{4 D \epsilon}+\frac{1}{4 \pi a D} \log \left(\frac{\epsilon}{a}\right)+\frac{n a}{2 D} I_{1}\right)\left(1-C_{\epsilon} \int_{\Omega} k(\mathbf{x}) e^{-\frac{\Phi(\mathbf{x})}{D}} d \mathbf{x}\right)=C_{\epsilon} n a^{2} I_{2}+O(n) .
$$

Thus we obtain to leading order

$$
C_{\epsilon}=\frac{\pi a+\epsilon \log \left(\frac{\epsilon}{a}\right)+2 n \pi a^{2} \epsilon I_{1}}{\left(\pi a+\epsilon \log \left(\frac{\epsilon}{a}\right)+2 n \pi a^{2} \epsilon I_{1}\right) \int_{\Omega} k(\mathbf{x}) e^{-\frac{\Phi(\mathbf{x})}{D}} d \mathbf{x}+4 \pi n a^{3} D \epsilon I_{2}} .
$$

To further compute for the probability $\langle P\rangle$ and the MFPT $\langle\tau\rangle$ using expression (69), we shall now consider two distributions of windows:

1. Random distribution,

2. Uniform distribution. 


\subsection{Effect of randomly distributed narrow windows located on a sphere on the arrival time}

When there $n \gg 1$ non-overlapping windows randomly distributed on the sphere, the probability distribution of windows is given by

$$
\rho(\phi, \theta)=\rho_{i}(\phi)=\frac{1}{4 \pi a^{2}} \mathbf{1}_{\left\{\phi>2 \arcsin \left(\frac{\epsilon}{a}\right)\right\}},
$$

for all $1 \leq i \leq n$. The condition $\left\{\phi>2 \arcsin \left(\frac{\epsilon}{a}\right)\right\}$ ensures non-overlapping. Using the change of variable $y=\sin \left(\frac{\phi}{2}\right)$, we re-write integral (65)

$$
I_{1}=\frac{1}{\pi a^{2}} \int_{\frac{\epsilon}{a}}^{1}\left(\frac{1}{y}+\log \left(\frac{y}{1+y}\right)\right) y d y=\frac{1}{2 \pi a^{2}}\left[x+\log (1+x)+x^{2} \log \left(\frac{x}{1+x}\right)\right]_{\frac{\epsilon}{a}}^{1},
$$

that is

$$
I_{1}=\frac{1}{2 \pi a^{2}}\left(1-2 \frac{\epsilon}{a}-\frac{\epsilon^{2}}{a^{2}} \log \left(\frac{\epsilon}{a}\right)\right) .
$$

In addition, we have for the second integral (66)

$$
I_{2}=\frac{1}{4 \pi a^{2}} \int_{0}^{2 \pi} \int_{2 \arcsin \left(\frac{\epsilon}{a}\right)}^{\pi} e^{-\frac{\Phi(\phi, \theta)}{D}} \sin (\phi) d \phi d \theta
$$

Replacing in equation (69), $I_{1}$ and $I_{2}$ by expressions (72) and (73), we obtain to leading order for randomly distributed windows,

$$
C_{\epsilon}^{\mathrm{rand}}=\frac{1}{\int_{\Omega} k(\mathbf{x}) e^{-\frac{\Phi(\mathbf{x})}{D}} d \mathbf{x}+C(n, \varepsilon) \int_{0}^{2 \pi} \int_{2 \frac{\epsilon}{a}}^{\pi} e^{-\frac{\Phi(\phi, \theta)}{D}} \sin (\phi) d \phi d \theta}
$$

where

$$
C(n, \varepsilon)=\frac{n a D \epsilon}{\pi a+\epsilon\left(1-\frac{n \epsilon^{2}}{a^{2}}\right) \log \left(\frac{\epsilon}{a}\right)+n \epsilon\left(1-2 \frac{\epsilon}{a}\right)} .
$$

\subsection{Effect of regularly distributed windows located on the sur- face $S_{a}$ on the mean time}

For small windows regularly distributed on a sphere, the density was computed in [7]:

$$
\rho(\phi)=\mathbf{1}_{\left\{\phi>\arccos \left(1-\frac{2}{n}\right)\right\} \frac{1}{4 \pi a^{2}}},
$$


leading to with the first integral (65) to

$$
I_{1}=\frac{1}{2 \pi a^{2}}\left[x+\log (1+x)+x^{2} \log \left(\frac{x}{1+x}\right)\right]_{\frac{1}{2} \arccos \left(1-\frac{2}{n}\right)}^{1},
$$

and for $n \gg 1$

$$
I_{1}=\frac{1}{2 \pi a^{2}}\left(1-\frac{2}{\sqrt{n}}+\frac{\log (n)}{2 n}\right)+o\left(\frac{\log (n)}{2 n}\right) \text {. }
$$

In addition, for second integral (66) we have

$$
I_{2}=\frac{1}{4 \pi a^{2}} \int_{0}^{2 \pi} \int_{2 \arccos \left(1-\frac{2}{n}\right)}^{\pi} e^{-\frac{\Phi(\phi, \theta)}{D}} \sin (\phi) d \phi d \theta
$$

Replacing in equation (69), $I_{1}$ and $I_{2}$ by expressions (78) and (79) respectively, we obtain

$$
C_{\epsilon}^{\text {hom }}=\frac{1}{\int_{\Omega} k(\mathbf{x}) e^{-\frac{\Phi(\mathbf{x})}{D}} d \mathbf{x}+\tilde{C}_{\epsilon, n} \int_{0}^{2 \pi} \int_{\frac{4}{\sqrt{n}}}^{\pi} e^{-\frac{\Phi(\phi, \theta)}{D}} \sin (\phi) d \phi d \theta} .
$$

where

$$
\tilde{C}_{\epsilon, n}=\frac{n a D \epsilon}{\pi a+\epsilon \log \left(\frac{\sqrt{n} \epsilon}{a}\right)+n \epsilon\left(1-\frac{2}{\sqrt{n}}\right)}
$$

Using formula (74) and (80) in expression (59), we obtain the asymptotic expressions for the probability and the condition MFPT that a stochastic particle reaches a small windows

$$
\langle P\rangle=\frac{F(n, a, \epsilon) \int_{0}^{2 \pi} \int_{\alpha_{0}}^{\pi} e^{-\frac{\Phi(\phi, \theta)}{D}} \sin (\phi) d \phi d \theta}{\int_{\Omega} k(\mathbf{x}) e^{-\frac{\Phi(\mathbf{x})}{D}} d \mathbf{x}+F(n, a, \epsilon) \int_{0}^{2 \pi} \int_{\alpha_{0}}^{\pi} e^{-\frac{\Phi(\phi, \theta)}{D}} \sin (\phi) d \phi d \theta}
$$

and

$$
\langle\tau\rangle=\frac{\int_{\Omega} e^{-\frac{\phi(\mathbf{x})}{D}} d \mathbf{x}}{\int_{\Omega} k(\mathbf{x}) e^{-\frac{\Phi(\mathbf{x})}{D}} d \mathbf{x}+F(n, a, \epsilon) \int_{0}^{2 \pi} \int_{\alpha_{0}}^{\pi} e^{-\frac{\Phi(\phi, \theta)}{D}} \sin (\phi) d \phi d \theta}
$$

where

$$
F(n, a, \epsilon)=\frac{n a D \epsilon}{\left(\pi a+\epsilon \log \left(\frac{\epsilon}{a}\right)+n \epsilon\left(1-2 \alpha_{0}-\alpha_{0}^{2} \log \left(\alpha_{0}\right)\right)\right)}
$$


and

$$
\alpha_{0}=\left\{\begin{array}{lc}
\frac{\epsilon}{a} & \text { for randomly distributed windows, } \\
\frac{1}{\sqrt{n}} & \text { for regularly distributed windows. }
\end{array}\right.
$$

When the drift is pointing towards the nucleus center and the potential $\Phi(\boldsymbol{x})=\Phi_{0}$ is constant at the nuclear surface, then the probability and MFPT formulas reduce to

$$
\langle P\rangle=\frac{4 \pi F(n, a, \epsilon) e^{-\frac{\Phi_{0}}{D}}}{\int_{\Omega} k(\mathbf{x}) e^{-\frac{\phi(\mathbf{x})}{D}} d \mathbf{x}+4 \pi F(n, a, \epsilon) e^{-\frac{\Phi_{0}}{D}}},
$$

and

$$
\langle\tau\rangle=\frac{\int_{\Omega} e^{-\frac{\phi(\mathbf{x})}{D}} d \mathbf{x}}{\int_{\Omega} k(\mathbf{x}) e^{-\frac{\phi(\mathbf{x})}{D}} d \mathbf{x}+4 \pi F(n, a, \epsilon) e^{-\frac{\Phi_{0}}{D}}} .
$$

When the drift $\Phi_{S_{a}}$ restricted to $S_{a}$ has a single global minima $\Phi_{m}$ at position $\mathbf{x}_{0}\left(\phi_{0}, \theta_{0}\right) \in$ $S_{a}$, we approximate integral $I_{2}$ using Laplace's method. In the small diffusion limit $D \ll \Phi(\mathbf{x})$ and large $\mathrm{n}$, we get

$$
I_{2}=\frac{1}{4 \pi a^{2}} \int_{0}^{2 \pi} \int_{2 \arccos \left(1-\frac{2}{n}\right)}^{\pi} e^{-\frac{\Phi(\phi, \theta)}{D}} \sin (\phi) d \phi d \theta \approx \frac{D}{4 a^{2} \sqrt{\operatorname{det}\left[-H_{\Phi_{S_{a}}}\left(\mathbf{x}_{0}\right)\right]}} e^{-\frac{\Phi_{m}}{D}}
$$

where det $\left[H_{\Phi_{S_{a}}}\left(\mathbf{x}_{0}\right)\right]$ is the determinant of the Hessian matrix of potential $\Phi_{S_{a}}$ at $\mathbf{x}_{0}$. The probability and MFPT to a nuclear pore are then given by

$$
\langle P\rangle=\frac{\pi D F(n, a, \epsilon) \sqrt{\operatorname{det}^{-1}\left[H_{\Phi_{S_{a}}}\left(\mathbf{x}_{0}\right)\right]} e^{-\frac{\Phi_{m}}{D}}}{\int_{\Omega} k(\mathbf{x}) e^{-\frac{\Phi(\mathbf{x})}{D}} d \mathbf{x}+\pi D F(n, a, \epsilon) \sqrt{\operatorname{det}^{-1}\left[H_{\Phi_{S_{a}}}\left(\mathbf{x}_{0}\right)\right]} e^{-\frac{\Phi_{m}}{D}}},
$$

and

$$
\langle\tau\rangle=\frac{\int_{\Omega} e^{-\frac{\phi(\mathbf{x})}{D}} d \mathbf{x}}{\int_{\Omega} k(\mathbf{x}) e^{-\frac{\Phi(\mathbf{x})}{D}} d \mathbf{x}+\pi D F(n, a, \epsilon) \sqrt{\operatorname{det}^{-1}\left[H_{\Phi_{S_{a}}}\left(\mathbf{x}_{0}\right)\right]} e^{-\frac{\Phi_{m}}{D}}} .
$$

A second Laplace's method can be used to estimate the volume integral. If the global minimum $\Phi_{\Omega}$ is attained at a point $\boldsymbol{x}_{g} \in \Omega$,

$$
\int_{\Omega} e^{-\frac{\phi(\mathbf{x})}{D}} d \mathbf{x} \approx \frac{(\pi D)^{3 / 2}}{\sqrt{\operatorname{det}\left[H_{\Phi}\left(\mathbf{x}_{g}\right)\right]}} e^{-\frac{\Phi_{\Omega}}{D}}
$$

We conclude this section by indicating that the formulas presented above can be used to estimate the probability and the mean time for a viral particle to reach a nuclear pore inside the nucleus. 


\subsection{Effect of changing the window coverage on the escape time}

For a large number of windows $n \gg 1$, distributed over a small target $S_{a}$ of a domain $\Omega$ and covering a proportion $\sigma=\frac{n \pi \epsilon^{2}}{\left|\partial S_{a}\right|}$ of the surface $\partial S_{a}$ of $S_{a}$, the leading order term in the NET for a Brownian particle to one of the small window was computed asymptotically using electrostatic consideration [25]

$$
\langle\tau\rangle_{E S}=\frac{|\Omega|}{D}\left(\frac{1}{C_{S_{a}}}+\frac{f(\sigma)}{4 n \epsilon}\right)
$$

where $|\Omega|$ is the volume, $C_{S_{a}}$ the capacity of the surface $\partial S_{a}$ containing the absorbing windows, and $f(\sigma)$ an unknown function that whose leading order is given by $f(\sigma)=1$ as $\sigma$ tends to 0 [3]. When the surface $S_{a}$ is a sphere of radius $a$, the capacitance $C_{S_{a}}=4 \pi a$, and the MFPT is given by

$$
\langle\tau\rangle_{E S}=\frac{|\Omega|}{D}\left(\frac{1}{4 \pi a}+\frac{f(\sigma)}{4 n \epsilon}\right)
$$

Here, for a Brownian particle (no drift and no killing measure), the MFPT (equation (83)) reduces to

$$
\langle\tau\rangle_{\Phi=0, k=0} \approx \frac{|\Omega|}{D}\left(\frac{1}{4 \pi a}+\frac{1}{4 n \epsilon}\left(1-\frac{n \epsilon}{\pi a}\left(2 \alpha_{0}-\alpha_{0}^{2} \log \left(\alpha_{0}\right)+\frac{1}{n} \log \left(\frac{\epsilon}{a}\right)\right)\right)\right) .
$$

Thus, we identify the function

$$
f(\sigma)=1-8 \frac{\sigma}{\pi}+\frac{\epsilon}{a \pi}(1-4 \sigma) \log \left(\frac{\epsilon}{a}\right)+o\left(\frac{\epsilon}{a}\right)
$$

when non-overlapping absorbing holes are randomly distributed and

$$
f(\sigma)=1-4 \frac{\sqrt{\sigma}}{\pi}+\frac{\epsilon}{a \pi} \log (\sqrt{\sigma})+o\left(\frac{\epsilon}{a}\right)
$$

when absorbing holes are regularly distributed (equally spaced). We end this section with two remarks. First, for a coverage $\sigma<<1,8 \frac{\sigma}{\pi}<4 \frac{\sqrt{\sigma}}{\pi}$ and the MFPT of a Brownian particle to an absorbing hole is larger for randomly compared to regularly distributed (equally spaced) holes. This regular distribution shall be close to the arrangement of Fekete points on a sphere that minimizes the MFPT [7]. Second, formulas (95) and (96) derived here by accounting for two window coverage predict that the MFPT formula is different than previously reported based on an effective approximation where $f(\sigma)=1-\sigma$ [30] or on an interpolation procedure using Brownian simulations for which $f(\sigma)=\frac{1-\sigma}{1+3.8 \sigma^{1.25}}[2]$. This latter difference may arise from the differences in the windows' arrangement. 


\section{Comparison of asymptotic formula with respect to Brownian simulations}

We compare our asymptotic formula for the probability (82) and conditional MFPT (83) with Monte-Carlo simulations when the number of holes $n$, their coverage of the nuclear surface $\sigma=\frac{n \pi \epsilon^{2}}{4 \pi a^{2}}$, or the distance $r_{0}$ between drift and cell centers vary. We model the cell as a ball of radius $R$ and the nucleus as a centered sphere $S_{a}$ (radius $a$ ) uniformly covered by $n$ small absorbing pores (radius $\epsilon$ ) (Figure 1 right). Stochastic particles are reflected on the external membrane $r=R$ and on $r=a$ except on all windows $\partial N_{a}=\bigcup_{i=1}^{n} \partial \Omega_{i}$, centered at random locations $\left(\mathbf{x}_{i}\right)_{i=1}^{n}$. To explore how the number of holes and their coverage impact the probability and conditional MFPT, we first use a constant radial drift $B$ directed toward the nucleus (with a potential $\Phi(r)=-B r$ ). We consider a constant killing rate $k(\mathbf{x})=k_{0}$ and consequently, using function

$$
G(D, B, a)=\frac{4 \pi e^{-\frac{B a}{D}}}{|\Omega|}\left(\frac{D}{B} a^{2}+2\left(\frac{D}{B}\right)^{2} a+2\left(\frac{D}{B}\right)^{3}\right)
$$

expressions (86-87) simplify to

$$
\langle P\rangle=\frac{e^{-\frac{B a}{D}}}{\langle\tau\rangle_{\Phi=0, k=0}(G(D, B, a)-G(D, B, R)) k+e^{-\frac{B a}{D}}},
$$

and

$$
\langle\tau\rangle=\frac{\langle\tau\rangle_{\Phi=0, k=0}(G(D, B, a)-G(D, B, R))}{\langle\tau\rangle_{\Phi=0, k=0}(G(D, B, a)-G(D, B, R)) k+e^{-\frac{B a}{D}}} .
$$

In Figure 2a, we compare these expressions to stochastic simulations for an increasing number of holes while maintaining constant the ratio $\sigma=\frac{n \pi \epsilon^{2}}{4 \pi a^{2}}=2 \%$ of the nucleus surface covered by the absorbing windows. This ratio was calibrated using a surface covered by 2,000 pores of $25 \mathrm{~nm}$ diameter on the nucleus of a chinese hamster ovary cell [22]) (parameters are summarized in table 1).

We evaluate the convergence of the Monte-Carlo simulations by computing the difference between two independent runs and stop the iteration when it is less than 5\%. The converge is attained for $N>10,000$ runs with a time step $d t<0.001 / \sqrt{n}$. These values show that Monte-Carlo simulations become rapidly intractable as the number of absorbing holes increases, confirming the need of asymptotic formula to analyse the passage time to many small holes. We limited our simulations to the range $1 \leq n \leq 30$. In this range, we observe that the asymptotic formula we derived match the simulation results. The remaining difference between the simulation results and the formula is 
probably due to higher order terms in asymptotic formula, that were recently discussed in [21]. Our analysis show that the conditional MFPT (respectively the probability) rapidly decreases (respectively increases) with the number of holes, the MFPT decreasing by $40 \%$ for $n=30$ (MFPT $=160 \mathrm{~s}$ ) compared to $n=1$ (MFPT $=270 s)$ and the probability being more than doubled ( $55 \%$ for $n=30$ compared to $26 \%$ for $n=1$ ). We also compare Monte-Carlo simulations to previous formula (11) that does not account for hole interactions $(f(\sigma)=0$ in expression (95)), and observe a significant mismatch even for few $(n>5)$ absorbing holes.

In a second ensemble of simulations, we kept constant the number of absorbing holes $(n=10)$ and we vary the coverage by absorbing windows $\sigma=\frac{n \epsilon^{2}}{a^{2}}$ from $2 \%$ to $50 \%$ (fig. 2b). We observe that formula (99) and (98) with the coverage function $f(\sigma)$ given by expression (95) match simulations even for high coverage $(\sigma \leq 30 \%)$ before a small difference appears. We also plotted MFPT and the probability with no correction for holes' coverage $(f(\sigma)=1)$, and observe that they deviate significantly from simulations for $\sigma \geq 5 \%$.

We also studied the conditional MFPT and probability for off-center drift. This question is particularly relevant in modeling viral trafficking as the vector field for the drift is centered at the centrosome, which close to the nucleus. We kept $n=10$ and $\sigma=2 \%$ and vary the distance $r_{0}$ between the domain and and the drift center from $r_{0}=0$ to $r_{0}=R=20 \mu m$ (Fig. 2c). The asymptotic formula (82) and (83) for the MFPT and the probability that a stochastic virus reaches an absorbing pore can be made more explicit by using a potential with an off-center constant drift $B$ equals to

$$
\Phi\left(r_{0}, r, \phi\right)=B \sqrt{r^{2}+r_{0}^{2}-2 r r_{0} \cos (\phi)} .
$$

Using this expression, the surface integral $\int_{0}^{\pi} e^{-\frac{\Phi\left(r_{0}, r, \phi\right)}{D}} \sin (\phi) d \phi$ and the volume integral $\int_{a}^{R} \int_{0}^{\pi} e^{-\frac{\Phi\left(r_{0}, r, \phi\right)}{D}} r^{2} \sin (\phi) d \phi d r$ in expressions (82) and (83) can be computed numerically.

These formula match the results of stochastic simulations in the entire interval of $r_{0} \in[0,20 \mu \mathrm{m}]$. The variance increases drastically with the distance $r_{0}$. This high variability is due to the random configuration of the $n=10$ holes that vary between simulations runs: if an absorbing window is located next to the drift center, the MFPT will be lower than the results obtained from an homogeneous distributed windows.

Finally, it has been hypothesized that large viruses characterized by small diffusion constant could be trapped to the centrosome and need to be propel actively to get back-transported toward the nucleus [4]. We thus plotted here the MFPT and the probability that a large virus with decreased diffusion $D^{\prime}=D / 10=0.13 \mu m^{2} s^{-1}$ reaches a nuclear pore with respect to the distance 
$r_{0}$ between the centrosome and the nucleus. We found that the probability decreases drastically for $r_{0} \geq 5$, and falls to approximately 0 for $r_{0} \geq 8 \mu \mathrm{m}$ suggesting that large viruses should use a different routing strategy, involving a combination of passive diffusion and retrograde transport to move from the centrosome toward the nucleus surface.

We conclude that Monte-Carlo simulations have shown that the asymptotic formula derived here are valid in large range of parameters. Moreover, the geometry of the cell, the nuclear pores configuration and the viral motion affect the ability of a single virus to reach a nuclear pore to deliver its genome.

\section{Conclusion}

We extended here the asymptotic formula for the NET and the probability that a stochastic viral particle reaches a nuclear pores originally developed in [12]. We also obtained a refinement of the classical Berg-Purcell formula for a Brownian particle to get absorbed on a partially reflected sphere.

The present approach applies to an intermittent dynamics with alternative periods of free diffusion and directed motion along MTs. This dynamics characterizes a large class of cellular transport. When the intermittent particle can be degraded (through the ubiquitin-proteasome machinery or trapped in the crowded cytoplasm), the asymptotical formula for the probability $\langle P\rangle$ and the mean time $\langle\tau\rangle$ to reach a small absorbing target among $n$ accounts for the geometrical interactions between the windows. When the targets co-localize on a small domain $S_{a}$, the asymptotics of $\langle P\rangle$ and $\langle\tau\rangle$ are derived in the

limit $\frac{\left|S_{a}\right|}{|\Omega|} \ll 1$. We confirmed the validity of asymptotics formula for the probability $\langle P\rangle$ and the mean time $\langle\tau\rangle$ respectively using Brownian simulations.

These formulas provide an estimate for the arrival time of DNA viruses to a small nuclear pore. Contrary to the classical narrow escape asymptotic where the leader order term contains most of the geometry, here the $O(1)$-term accounts for the interactions between windows, discussed in $[24,6]$. For viruses, where the number of nuclear pores is very high $(n \approx 2,000, \sigma=2 \%[22])$ making Brownian simulations intractable, our formula predict that $\langle\tau\rangle=90$ seconds and $\langle P\rangle=74 \%$ which is close to the MFPT $\langle\tau\rangle=78$ seconds and the probability $\langle P\rangle=78 \%$ to reach a completely absorbing nucleus. Moreover, these estimates are far from the ones derived in [12] with formula (11) for a single big pore covering $\sigma=2 \%$ of the nucleus $(\langle\tau\rangle=268$ seconds and $\langle P\rangle=26 \%)$. This shows that the fragmentation of absorbing targets in many smaller targets renders the overall absorption process much more efficient.

Finally, MTs along which viruses are transported point towards the centrosome which is close to the nucleus leading to an off-center effective potential. Our formula shows that the distance of drift to cell center decreases the prob- 


\section{A- Number of pores}
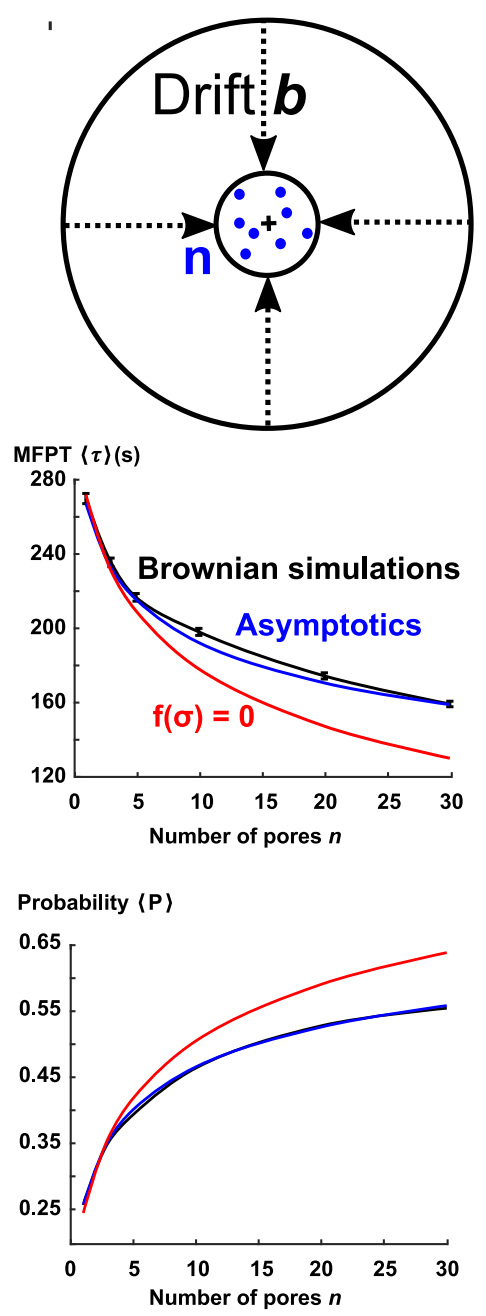

\section{B- Coverage}
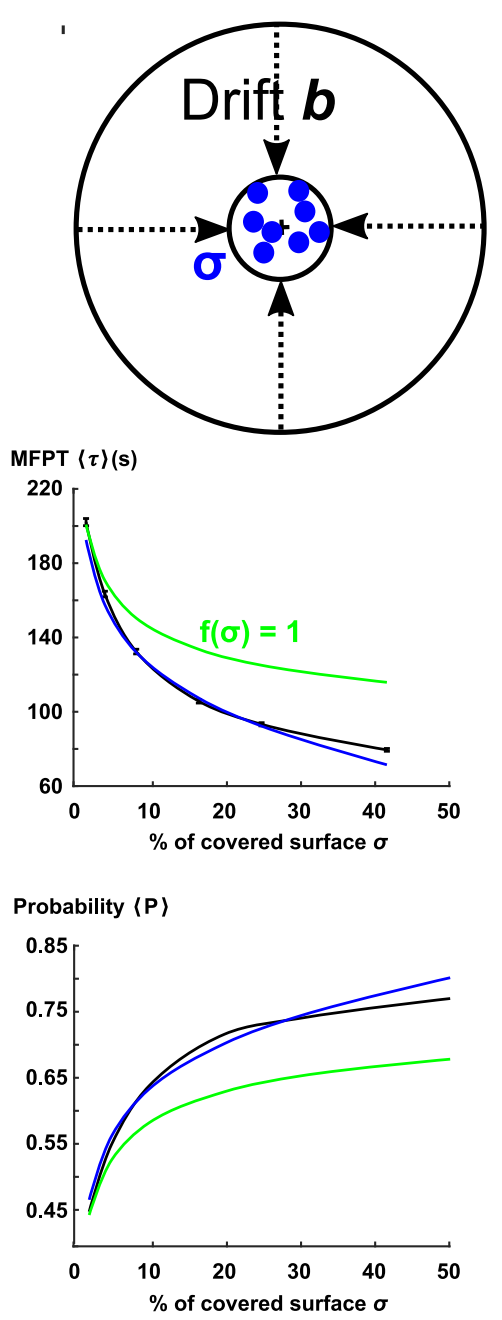

\section{C- Off-center drift}

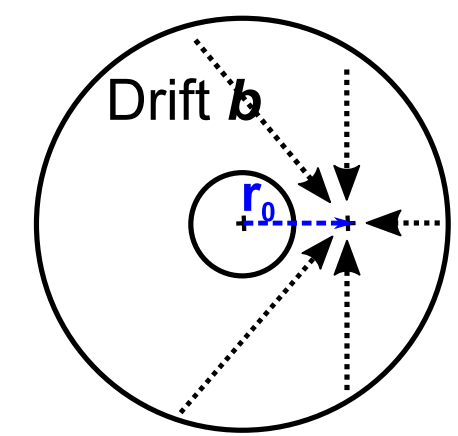

MFPT $\langle\tau\rangle(\mathbf{s})$

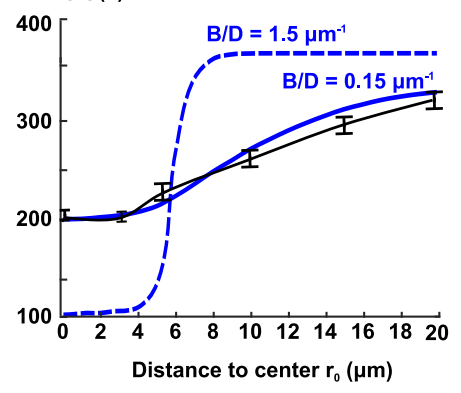

Probability $\langle\mathbf{P}\rangle$

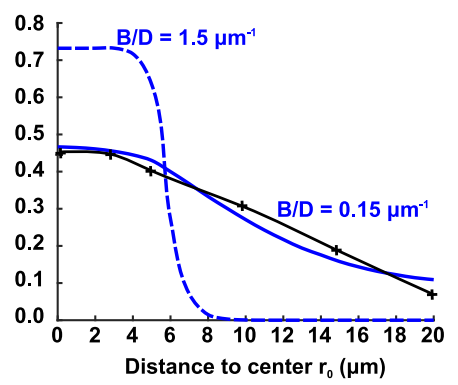

Figure 2: Conditional MFPT $\langle\tau\rangle$ and probability $\langle P\rangle$ for a virus to reach an absorbing nuclear pore. a- Centered drift, absorbing surface coverage ratio $\sigma=2 \%$. For a centered drift, the MFPT (equation (99)) and probability (equation (98)) formula (blue) is compared to stochastic simulations (black, bars $= \pm 1$ standard error) for an increasing number of absorbing windows, distributed randomly. The coverage ratio $\sigma=\frac{n \pi \epsilon^{2}}{4 \pi a^{2}}$ of absorbing windows to nuclear surface is fixed to $\sigma=2 \%$ [22]. Plot of the asymptotics formula (9) (corresponding to $f(\sigma)=0$ in equation (95)) that neglects windows interactions (red). b- Centered drift, number of pores $n=10$ fixed. Same as in a, but now the number of random, absorbing pores is fixed to $n=10$. We plot the formula without coverage correction $(f(\sigma)=1$ in equation (95)). c- Non-centered drift, coverage $\sigma=2 \%$ and number of pores $n=10$ fixed. We shifted the drift-center at a distance $0 \leq r_{0} \leq R$ from the center and compare MFPT (83) and probability (82) as in $\mathbf{a}$. The coverage ratio $\sigma=2 \%$ and the number of pores $n=10$ are kept constant. The asymptotic formula for a smaller diffusion $D^{\prime}=0.13 \mu \mathrm{m}^{2} \mathrm{~s}^{-1}=D / 10$ and a ratio $B / D^{\prime}=1.5 \mu m^{-1}$ are shown (dashed blue). We used 10000 stochastic trajectories with a time step $d t=0.001 / \sqrt{n}$. Parameters are summarized in table 1 . 
ability that a virus reaches a nuclear pore before being degraded, and that large viruses with small diffusion coefficient are even more affected. Indeed, for a distance $r_{0}=6 \mu \mathrm{m}$ just above the nucleus radius $a=5 \mu \mathrm{m}$, our formula predicts that the viral probability $\langle P\rangle$ is decreased to $56 \%$, and falls to $4.8 \%$ and $0.0004 \%$ when the diffusion coefficient is divided by $10\left(D=0.13 \mu \mathbf{m}^{2} \mathbf{s}^{-1}\right)$ or 100 $\left(D=0.013 \mu \mathbf{m}^{2} \mathbf{s}^{-1}\right)$. The latter small diffusion coefficient has been reported for many large viruses (table 1 in [29]), and other mechanisms such as retrograde transport of viruses from the centrosome to the nucleus might be involved in viral trafficking to optimize the delivery of their genome to the nucleus.

It would certainly be valuable to extend the present work by considering the exact mechanism, where large molecules are being imported through nuclear pores. Such model should account for cargo molecules that transport large molecules though the pore, which is an energy costly process (ATPdependent). Such model should be further coarse-grained into a steady-state Robin boundary condition, instead of using the pure absorbing condition at the windows, as we did here. The constant in the Robin term should then be carefully derived from the model equations, see also [21].

\begin{tabular}{|c|c|c|}
\hline Parameter & Description & Value \\
\hline$D$ & Diffusion constant of the virus & $\begin{array}{l}D=1.3 \mu m^{2} s^{-1} \\
\text { for the Associated-Adeno-Virus [27] }\end{array}$ \\
\hline$B$ & Drift & $B=0.2 \mu m s^{-1}[17]$ \\
\hline$\sigma$ & $\begin{array}{l}\% \text { of the nuclear surface covered } \\
\text { by } n \text { nuclear pores }\end{array}$ & $\sigma=2 \%[22]$ \\
\hline$k$ & Degradation rate & $\begin{array}{l}k=1 / 360 s^{-1} \text { (10 times the rate observed } \\
\text { for gene vectors }[20])\end{array}$ \\
\hline$R$ & Radius of the cell & $R=20 \mu m($ Chinese hamster ovary cell $)$ \\
\hline$a$ & Radius of the nucleus & $a=5 \mu m[22]$ \\
\hline
\end{tabular}

Table 1: Numerical parameters used for Brownian simulations

\section{Appendix}

We derive in this appendix the asymptotic of the Neumann's function $\mathcal{N}\left(\boldsymbol{x}_{i}, \boldsymbol{x}_{j}\right)$ for two absorbing patches of size $\varepsilon$ located on the surface of a small ball of radius $a$. Expansion (48) is insufficient and we shall now derive a two parameter expansion in $a$ and $\varepsilon$. We start by observing that the $\log$-term $\frac{-1}{4 \pi a D} \log \left(\frac{1}{\left|\boldsymbol{x}_{i}-\boldsymbol{x}_{j}\right|}\right)$ can be much larger than the leading 
order term $\frac{1}{2 \pi D\left|\boldsymbol{x}_{i}-\boldsymbol{x}_{j}\right|}$ when $\left|\boldsymbol{x}_{i}-\boldsymbol{x}_{j}\right| \approx a$, for $a \ll|\Omega|^{\frac{1}{3}}$. Consequently, we shall reexamine the $\log$-term expansion. For $\boldsymbol{x}$ and $\boldsymbol{x}_{0}$ in the neighborhood of the sphere $S_{a}$, we expand the Neumann function $\mathcal{N}\left(\boldsymbol{x}, \boldsymbol{x}_{0}\right)$

$$
\mathcal{N}\left(\boldsymbol{x}, \boldsymbol{x}_{0}\right)=\tilde{\mathcal{N}}\left(\boldsymbol{x}, \boldsymbol{x}_{0}\right)+O(1)
$$

where $\tilde{\mathcal{N}}\left(\boldsymbol{x}, \boldsymbol{x}_{0}\right)$ is solution with $D=1$ of the Laplace equation

$$
\begin{aligned}
& \Delta \tilde{\mathcal{N}}\left(\boldsymbol{x}, \boldsymbol{x}_{0}\right)=-\delta\left(\boldsymbol{x}-\boldsymbol{x}_{0}\right) \text { for } \boldsymbol{x} \in \mathbb{R}^{3} \\
& \frac{\partial \tilde{\mathcal{N}}}{\partial n}\left(\boldsymbol{x}, \boldsymbol{x}_{0}\right)=0 \text { for } \boldsymbol{x} \in S_{a} .
\end{aligned}
$$

To compute the log-term, we first decompose $\tilde{\mathcal{N}}\left(\boldsymbol{x}, \boldsymbol{x}_{0}\right)=\frac{1}{4 \pi\left|\boldsymbol{x}-\boldsymbol{x}_{0}\right|}+\Phi\left(\boldsymbol{x}, \boldsymbol{x}_{0}\right)$ where $\Phi$ is solution of the system:

$$
\begin{aligned}
& \Delta \Phi\left(\boldsymbol{x}, \boldsymbol{x}_{0}\right)=0, \text { for } \boldsymbol{x} \in \mathbb{R}^{3} \\
& \frac{\partial \Phi}{\partial n}\left(\boldsymbol{x}, \boldsymbol{x}_{0}\right)=-\frac{\partial}{\partial n}\left(\frac{1}{4 \pi\left|\boldsymbol{x}-\boldsymbol{x}_{0}\right|}\right), \text { for } \boldsymbol{x} \in S_{a} .
\end{aligned}
$$

To solve equation (103), we choose a coordinate system for which the point source $\boldsymbol{x}=\boldsymbol{x}_{0}$ is on the positive $z$-axis. Since $a \Phi=0$ and $\Phi$ is axisymmetric, it has the series expansion

$$
\Phi\left(\boldsymbol{x}, \boldsymbol{x}_{0}\right)=\sum_{n=0}^{\infty} b_{n}\left(\left|\boldsymbol{x}_{0}\right|\right) \frac{P_{n}(\cos (\theta))}{|\boldsymbol{x}|^{n+1}}
$$

where $P_{n}$ are the Legendre polynomials of integer $n, \theta$ is the angle between $\boldsymbol{x}$ and the north pole and $b_{n}\left(\left|\boldsymbol{x}_{0}\right|\right)$ are coefficients, determined from boundary condition (103). For $\boldsymbol{x} \in S_{a}$ and $\rho=|\boldsymbol{x}|$,

$$
\frac{\partial \Phi}{\partial n}\left(\boldsymbol{x}, \boldsymbol{x}_{0}\right)=\frac{\partial \Phi}{\partial \rho}(\rho=a)=-\sum_{n=0}^{\infty} \frac{(n+1) b_{n}\left(\left|\boldsymbol{x}_{0}\right|\right)}{a^{n+2}} P_{n}(\cos (\theta) .
$$

For $|\boldsymbol{x}|<\left|\boldsymbol{x}_{0}\right|$, we have the expansion

$$
\frac{1}{4 \pi\left|\boldsymbol{x}-\boldsymbol{x}_{0}\right|}=\frac{1}{4 \pi} \sum_{n=0}^{\infty} \frac{|\boldsymbol{x}|^{n}}{\left|\boldsymbol{x}_{0}\right|^{n+1}} P_{n}(\cos (\theta))
$$

which leads to the boundary condition:

$$
-\frac{\partial}{\partial \rho}\left(\frac{1}{4 \pi\left|\boldsymbol{x}-\boldsymbol{x}_{0}\right|}\right)(\rho=a)=-\frac{1}{4 \pi} \sum_{n=0}^{\infty} \frac{n a^{n-1}}{\left|\boldsymbol{x}_{0}\right|^{n+1}} P_{n}(\cos (\theta)) .
$$


Injecting relations (105-107) into the boundary condition (103), we obtain that for all $n \geq 0$ :

$$
b_{n}\left(\left|\boldsymbol{x}_{0}\right|\right)=\frac{1}{4 \pi} \frac{n a^{2 n+1}}{(n+1)\left|\boldsymbol{x}_{0}\right|^{n+1}} .
$$

The Neumann function $\tilde{\mathcal{N}}\left(\boldsymbol{x}, \boldsymbol{x}_{0}\right)$ is then given by:

$$
\tilde{\mathcal{N}}\left(\boldsymbol{x}, \boldsymbol{x}_{0}\right)=\frac{1}{4 \pi\left|\boldsymbol{x}-\boldsymbol{x}_{0}\right|}+\frac{1}{4 \pi} \sum_{n=0}^{\infty} \frac{n a^{2 n+1}}{(n+1)|\boldsymbol{x}|^{n+1}\left|\boldsymbol{x}_{0}\right|^{n+1}} P_{n}(\cos (\theta)),
$$

that we rewrite

$\tilde{\mathcal{N}}\left(\boldsymbol{x}, \boldsymbol{x}_{0}\right)=\frac{1}{4 \pi\left|\boldsymbol{x}-\boldsymbol{x}_{0}\right|}+\frac{1}{4 \pi} \sum_{n=0}^{\infty}\left(\frac{a^{2 n+1}}{|\boldsymbol{x}|^{n+1}\left|\boldsymbol{x}_{0}\right|^{n+1}}-\frac{a^{2 n+1}}{(n+1)|\boldsymbol{x}|^{n+1}\left|\boldsymbol{x}_{0}\right|^{n+1}}\right) P_{n}(\cos (\theta))$

Using expansion (106), we have for the first term of (110),

$$
\frac{1}{4 \pi} \sum_{n=0}^{\infty} \frac{a^{2 n+1}}{|\boldsymbol{x}|^{n+1}\left|\boldsymbol{x}_{0}\right|^{n+1}} P_{n}(\cos (\theta))=\frac{a}{4 \pi\left|\boldsymbol{x}_{0}\right| \mid x-\frac{a^{2} \boldsymbol{x}_{0} \mid}{\left|\boldsymbol{x}_{0}\right|^{2}}}
$$

To compute the second term $I(\rho)=-\sum_{n=0}^{\infty} \frac{a^{2 n+1}}{(n+1) \rho^{n+1}\left|\boldsymbol{x}_{0}\right|^{n+1}} P_{n}(\cos (\theta))$, we differentiate

$$
I^{\prime}(\rho)=\sum_{n=0}^{\infty} \frac{a^{2 n+1}}{\rho^{n+2}\left|\boldsymbol{x}_{0}\right|^{n+1}} P_{n}(\cos (\theta))=\frac{a}{\rho\left|\boldsymbol{x}_{0}\right| \mid x-\frac{a^{2} \boldsymbol{x}_{0} \mid}{\left|\boldsymbol{x}_{0}\right|^{2}}}
$$

that is

$$
I^{\prime}(\rho)=\frac{1}{\rho a\left(1+\frac{\left|\boldsymbol{x}_{0}\right|^{2} \rho^{2}}{a^{4}}-2 \frac{\left|\boldsymbol{x}_{0}\right| \rho}{a^{2}} \cos (\theta)\right)^{\frac{1}{2}}} .
$$

Because $\lim _{\rho \rightarrow \infty} l(\rho)=0$, we have:

$$
l(\rho)=-\int_{\rho}^{\infty} I^{\prime}(s) d s=-\int_{\rho}^{\infty} \frac{d s}{s a\left(1+\frac{\left|\boldsymbol{x}_{0}\right|^{2} s^{2}}{a^{4}}-2 \frac{\left|\boldsymbol{x}_{0}\right| s}{a^{2}} \cos (\theta)\right)^{\frac{1}{2}}} .
$$

Thus,

$$
l(\rho)=\frac{1}{a} \log \left(\frac{\frac{\left|\boldsymbol{x}_{0}\right| \rho}{a^{2}}(1-\cos (\theta))}{1-\frac{\left|\boldsymbol{x}_{0}\right| \rho}{a^{2}} \cos (\theta)+\left(1+\left(\frac{\left|\boldsymbol{x}_{0}\right| \rho}{a^{2}}\right)^{2}-2 \frac{\left|\boldsymbol{x}_{0}\right| \rho}{a^{2}} \cos (\theta)\right)^{\frac{1}{2}}}\right)
$$


Finally, we obtain the expression of the Neumann function $\tilde{\mathcal{N}}\left(\boldsymbol{x}, \boldsymbol{x}_{0}\right)$ and the exact dependency with respect to the inner ball of radius $a$ :

$$
\begin{aligned}
\tilde{\mathcal{N}}\left(\boldsymbol{x}, \boldsymbol{x}_{0}\right) & =\frac{1}{4 \pi\left|\boldsymbol{x}-\boldsymbol{x}_{0}\right|}+\frac{a}{4 \pi D\left|\boldsymbol{x}_{0}\right| \mid x-\frac{a^{2} \boldsymbol{x}_{0} \mid}{\left|\boldsymbol{x}_{0}\right|^{2}}} \\
& +\frac{1}{4 \pi a} \log \left(\frac{\frac{\left|\boldsymbol{x}_{0}\right||\boldsymbol{x}|}{a^{2}}(1-\cos (\theta))}{1-\frac{\left|\boldsymbol{x}_{0}\right||\boldsymbol{x}|}{a^{2}} \cos (\theta)+\left(1+\left(\frac{\left|\boldsymbol{x}_{0}\right||\boldsymbol{x}|}{a^{2}}\right)^{2}-2 \frac{\left|\boldsymbol{x}_{0} \| \boldsymbol{x}\right|}{a^{2}} \cos (\theta)\right)^{\frac{1}{2}}}\right)
\end{aligned}
$$

When $\boldsymbol{x}$ and $\boldsymbol{x}_{0}$ are on the sphere $S_{a},\left|\boldsymbol{x}_{0}\right|=|\boldsymbol{x}|=a$, we have

$$
\tilde{\mathcal{N}}\left(\boldsymbol{x}, \boldsymbol{x}_{0}\right)=\frac{1}{2 \pi\left|\boldsymbol{x}-\boldsymbol{x}_{0}\right|}+\frac{1}{4 \pi a} \log \left(\frac{\left|\boldsymbol{x}-\boldsymbol{x}_{0}\right|}{2 a+\left|\boldsymbol{x}-\boldsymbol{x}_{0}\right|}\right) .
$$

Acknowledgments: T.L. is supported by a FRM post-doctoral fellowship and a grant from Philippe Foundation. D. H. research is supported by a Marie-Curie fellowship.

\section{References}

[1] Arhel, N., Genovesio, A., Kim, K.A., Miko, S., Perret, E., Olivo-Marin, J.C., Shorte, S., Charneau, P.: Quantitative four-dimensional tracking of cytoplasmic and nuclear hiv-1 complexes. Nat Methods 3(10), 817-24 (2006). DOI 10.1038/nmeth928

[2] Berezhkovskii, A.M., Makhnovskii, Y.A., Monine, M.I., Zitserman, V.Y., Shvartsman, S.Y.: Boundary homogenization for trapping by patchy surfaces. J Chem Phys 121(22), 11,390-4 (2004). DOI 10.1063/1.1814351

[3] Berg, H.C., Purcell, E.M.: Physics of chemoreception. Biophys J 20(2), 193-219 (1977). DOI 10.1016/S0006-3495(77)85544-6

[4] Burckhardt, C.J., Suomalainen, M., Schoenenberger, P., Boucke, K., Hemmi, S., Greber, U.F.: Drifting motions of the adenovirus receptor car and immobile integrins initiate virus uncoating and membrane lytic protein exposure. Cell Host Microbe 10(2), 105-17 (2011). DOI 10.1016/j.chom.2011.07.006

[5] Chevalier, C., Bénichou, O., Meyer, B., Voituriez, R.: First-passage quantities of brownian motion in a bounded domain with multiple targets: a unified approach. Journal of Physics A: Mathematical and Theoretical 44, 025,002 (2011)

[6] Cheviakov, A.F., Ward, M., Straube, R.: An asymptotic analysis of the mean first passage time for narrow escape problems: Part ii: The sphere. SIAM Multiscale Modeling and Simulation 8(3), 836-870 (2010) 
[7] Cheviakov, A.F., Zawada, D.: Narrow-escape problem for the unit sphere: homogenization limit, optimal arrangements of large numbers of traps, and the n(2) conjecture. Phys Rev E Stat Nonlin Soft Matter Phys 87(4), 042,118 (2013). DOI 10.1103/PhysRevE.87.042118

[8] Coombs, D., Straube, R., Ward, M.: Diffusion on a sphere with localized traps: Mean first passage time, eigenvalue asymptotics, and fekete points. SIAM Journal On Applied Mathematics 70(1), 302-332 (2009)

[9] Dauty, E., Verkman, A.S.: Actin cytoskeleton as the principal determinant of sizedependent dna mobility in cytoplasm: a new barrier for non-viral gene delivery. J Biol Chem 280(9), 7823-8 (2005). DOI 10.1074/jbc.M412374200

[10] Dynes, J.L., Steward, O.: Dynamics of bidirectional transport of arc mrna in neuronal dendrites. J Comp Neurol 500(3), 433-47 (2007). DOI 10.1002/cne.21189

[11] Greber, U.F., Way, M.: A superhighway to virus infection. Cell 124(4), 741-54 (2006). DOI 10.1016/j.cell.2006.02.018

[12] Holcman, D.: Modeling dna and virus trafficking in the cell cytoplasm. Journal of Statistical Physics 127(3), 471-494 (2007)

[13] Holcman, D., Marchewka, A., Schuss, Z.: Survival probability of diffusion with trapping in cellular neurobiology. Phys Rev E Stat Nonlin Soft Matter Phys 72(3 Pt 1), 031,910 (2005). DOI 10.1103/PhysRevE.72.031910

[14] Holcman, D., Schuss, Z.: Escape through a small opening: Receptor trafficking in a synaptic membrane. Journal of Statistical Physics 117(5), 975-1014 (2004)

[15] Holcman, D., Schuss, Z.: Diffusion through a cluster of small windows and flux regulation in microdomains. Physics Letters A 372(21), 3768-3772 (2008)

[16] Holcman, D., Schuss, Z.: Stochastic Narrow Escape in Molecular and Cellular Biology: Analysis and Applications. Springer (2015)

[17] Lagache, T., Dauty, E., Holcman, D.: Quantitative analysis of virus and plasmid trafficking in cells. Phys Rev E Stat Nonlin Soft Matter Phys 79(1 Pt 1), 011,921 (2009). DOI 10.1103/PhysRevE.79.011921

[18] Lagache, T., Holcman, D.: Effective motion of a virus trafficking inside a biological cell. Siam Journal On Applied Mathematics 68(4), 1146-1167 (2008)

[19] Lagache, T., Holcman, D.: Quantifying intermittent transport in cell cytoplasm. Phys Rev E Stat Nonlin Soft Matter Phys 77(3 Pt 1), 030,901 (2008). DOI 10.1103/PhysRevE.77.030901 
[20] Lechardeur, D., Sohn, K.J., Haardt, M., Joshi, P.B., Monck, M., Graham, R.W., Beatty, B., Squire, J., O'Brodovich, H., Lukacs, G.L.: Metabolic instability of plasmid dna in the cytosol: a potential barrier to gene transfer. Gene Ther 6(4), 482-97 (1999). DOI 10.1038/sj.gt.3300867

[21] Lindsay, A., Ward, M.: First passage statistics for the capture of a brownian particle by a structured spherical target with multiple surface traps. ArXiv (2016.)

[22] Maul, G.G., Deaven, L.: Quantitative determination of nuclear pore complexes in cycling cells with differing dna content. J Cell Biol 73(3), 748-60 (1977)

[23] Medalia, O., Weber, I., Frangakis, A.S., Nicastro, D., Gerisch, G., Baumeister, W.: Macromolecular architecture in eukaryotic cells visualized by cryoelectron tomography. Science 298(5596), 1209-13 (2002). DOI 10.1126/science.1076184

[24] Pillay, S., Ward, M., Pierce, A., Kolokolnikov, T.: An asymptotic analysos of the mean first passage time for narrow escape problems: Part i: Two-dimensional domains. SIAM Multiscale Modeling and Simulation 8(3), 803-835 (2010)

[25] Reingruber, J., Abad, E., Holcman, D.: Narrow escape time to a structured target located on the boundary of a microdomain. J Chem Phys 130(9), 094,909 (2009). DOI 10.1063/1.3081633

[26] Schuss, Z., Holcman, D.: Diffusion escape through a cluster of small absorbing windows. Journal of Physics A: Mathematical and Theoretical 41(15), 155,001 (2008)

[27] Seisenberger, G., Ried, M.U., Endress, T., Büning, H., Hallek, M., Bräuchle, C.: Real-time single-molecule imaging of the infection pathway of an adeno-associated virus. Science 294(5548), 1929-32 (2001). DOI 10.1126/science.1064103

[28] Singer, A., Schuss, Z., Holcman, D.: Narrow escape and leakage of brownian particles. Phys Rev E Stat Nonlin Soft Matter Phys 78(5 Pt 1), 051,111 (2008). DOI 10.1103/PhysRevE.78.051111

[29] Sodeik, B.: Mechanisms of viral transport in the cytoplasm. Trends Microbiol 8(10), 465-72 (2000)

[30] Zwanzig, R.: Diffusion-controlled ligand binding to spheres partially covered by receptors: an effective medium treatment. Proc Natl Acad Sci U S A 87(15), 5856-7 (1990) 